\title{
Estilos gerenciales y su influencia en la generación de valor de las Instituciones Prestadoras de Salud de la Región Caribe ${ }^{1}$
}

\section{Management styles and their influence on value generation of the health care institutions of the Caribbean region}

DOI: http://dx.doi.org/10.17981/econcuc.38.1.06

Hugo Hernández Palma²

Delvis Muñoz Rojas ${ }^{3}$

Ignacio Barrios Parejo ${ }^{4}$

\section{Resumen}

En la actualidad, la generación de valor agregado ha sobrepasado los límites económicos para concebirse en el ámbito empresarial como una práctica integral en la cual intervienen las instituciones prestadoras de salud IPS. Es por ello, que se plantea el objetivo de determinar cómo los estilos y habilidades gerenciales influyen en la generación de valor en las IPS de la región Caribe. Para los efectos, se estableció una investigación de tipo descriptiva-documental con método cualitativo. La técnica seleccionada fue la entrevista estructurada que permitió la interacción con los gerentes de doce IPS. Se determinó que en el contexto de estudio prevalecen importantes debilidades que afectan la generación de valor en la gestión de las IPS y, en consecuencia, se enfrentan a riesgos y se minimiza el aprovechamiento de las oportunidades que surgen en ambientes dinámicos. Se concluye la necesidad de fortalecer la capacidad de liderazgo en los gerentes como factor determinante para optimar la gestión que impulse la generación de valor en procesos relacionados con empleados, clientes, proveedores, accionistas y sectores relacionados.

Palabras clave: estilos gerenciales; habilidades gerenciales; generación de valor; gestión de calidad.

Recibido: 21/11/2017 Devuelto para revisión: 23/5/17 Aceptado: 30/5/2017.

\begin{abstract}
${ }^{1}$ Artículo derivado del Proyecto de Investigación titulado: Gestión de proyecto estratégico de calidad para instituciones prestadoras de servicios de salud en el departamento del Atlántico para una mejora continua y consecución de la competitividad. Registrado bajo el Nro. CEO 19-COD2015. ADSCRITO AL Grupo de investigación INVESCARIBE DEL Programa de Administración de Empresas de la Universidad del Atlántico.

${ }^{2}$ Magister en Sistemas de Gestión. Universidad Autónoma del Caribe Ingeniero Industrial. Universidad Antonio Nariño. Universidad del Atlántico. Profesor e Investigador de la Universidad del Atlántico. Colombia hugohernandez@gmail.com

${ }^{3}$ Magister en Sistemas de Gestión. Psicóloga. Profesora de la Universidad de la Guajira. Colombia delvis21mr@gmail.com

${ }^{4}$ Doctor en Ciencias Gerenciales. Universidad Rafael Belloso Chacín. Venezuela. Administrador de Empresas. Profesor de la Universidad del Atlántico. Colombia. profesorignaciobarrios@hotmail.com
\end{abstract}

\author{
- The author; licensee Universidad de la Costa - CUC. \\ Económicas CUC vol. 38 no. 1, pp. 133-146. Enero - Junio, 2017 \\ Barranquilla. ISSN 0120-3932 Impreso, ISSN 2382-3860 Online
}

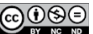




\begin{abstract}
Nowadays, the value generation has exceeded the economic limits to build in the business context fundamental practices which the health care providers are involving, in other words IPS. The purpose of the present work is to determine the influence of managerial styles and competences on value generation in the health sector of the Caribbean Region. With regard to the type of research, techniques and procedures, a descriptive documentary through qualitative methods as well interactive structured interviews were used, the chosen sample were managers of twelve IPS. The obtained results have showed that in the mentioned institutions there exist relevant lacks that affect the management of the IPS consequently, they have coped with several risks which have reduced their opportunities to expanding into this market. It can be said that the need to strengthen the leadership capacity in managers is as a determinant factor to enhance processes related to employees, customers, suppliers, shareholders and associated sectors..
\end{abstract}

Key words: managerial styles, managerial skills, value generation, quality management.

\title{
Introducción
}

El sistema de seguridad de salud en Colombia fue regulado con la ley 100 de 1993, la cual estableció las normas para el desarrollo de la prestación del servicio en salud y de igual forma instauró a la Superintendencia de Servicios Públicos (Supersalud), como el ente orgánico encargado de vigilar y controlar las IPS, entre otras (MPS, 2011). De acuerdo con la Ley mencionada, se especifican dos regímenes, bajo los cuales han de quedar amparados todos los colombianos. El primer régimen corresponde al Subsidiado, al cual se afilia la población con menos recursos y sin capacidad de pago.

El referido régimen es subvencionado en su totalidad por el Estado, y a través del Sistema de Selección de Beneficiarios para Programas Sociales (Sisben), se realiza la escogencia de 
los ciudadanos que reúnen las condiciones para afiliarse a este sistema de salud subsidiado y dirigir de eta forma el gasto público a las poblaciones más vulnerables. El segundo régimen denominado Contributivo, es aquel al cual se afilia la población vinculada laboralmente o con capacidad de pago como los trabajadores independientes y pensionados. Esta población se afilia al régimen de salud a través de la Entidades Promotoras de Salud (EPS), las cuales deben contar con una red de clínicas, hospitales o centros médicos para la prestación de los servicios de salud.

Estos centros de atención médica son las denominadas Instituciones Prestadoras de Salud (IPS), por medio de las cuales, los usuarios tienen acceso al sistema de salud, previa autorización de las Entidades Prestadoras de Salud. Es así, como las IPS prestan los servicios y realizan los respectivos cobros a las EPS, quienes a su vez están en la obligación de reembolsar los dineros cobrados por las IPS (MPS, 2010). Según lo expuesto, la crisis económica que actualmente afectan algunas Entidades Prestadoras de Salud, ha incidido en la situación actual de las IPS, a pesar de constituirse en un soporte fundamental para el bienestar social de las poblaciones asentadas en la región caribe colombiana.

Sin embargo, a pesar de su función principal, prevalecen importantes debilidades, como son las frecuentes crisis en la gestión organizacional y de servicios, así como declives económicos propios del sistema de salud. Asimismo, se hace referencia a la falta de financiación por parte del Estado; situaciones que demanda importantes retos para los líderes de las IPS, que aspiran permanecer y posicionarse en el mercado. En función de ello, se destaca la importancia de desarrollar investigaciones que redunden en la generación de aportes inherentes con el liderazgo de los gerentes de las referidas instituciones objeto de estudio.

En tal sentido, se define el objetivo de determinar cómo los estilos y habilidades gerenciales influyen en la generación de valor en las IPS de la región Caribe. Para lo cual, se desarrolla la estructura de contenido basado en tres partes fundamentales: en primera instancia, se estudian los estilos gerenciales y el perfil de los lideres contemporáneos, además se identificarán las habilidades, destrezas, aptitudes y actitudes que deben caracterizar el estilo del gerente para, a partir de ello, determinar cómo inciden estas características particulares en el manejo de recursos, la gestión de calidad y la generación de valor agregado en los resultados de las entidades, es decir, en la eficiencia y eficacia de las IPS de la región Caribe. Una segunda parte donde ese expone la ruta metodológica que enmarca la investigación, y por último se presenta los resultados y aspectos concluyentes. 


\section{Fundamentos sobre los estilos gerenciales}

El proceso de revisión documental, permitió determinar que se conciben diez tipos de estilos gerenciales, de los cuales su vigencia se condiciona a la capacidad e intereses de los gerentes en practicar de acuerdo al conjunto de actitudes y aptitudes consolidadas. Según Ruiz (2012) dadas las exigencias de los nuevos modelos administrativos, exponen seis estilos gerenciales, que mayor impacto genera en el contexto de las organizaciones objeto de estudio, se destacan:

La gerencia autocrático: según Solys (2016) es aquel que hace énfasis en la disciplina, la obediencia y la eficacia en el cumplimiento de metas, de manera rápida, estableciendo una clara jerarquía con sus colaboradores, así pues, este tipo de gerente lleva una vida muy activa, le cuesta mantenerse quieto y tiende incluso a ser operativo; como es una persona tan impulsiva, difícilmente escucha de manera asertiva las opiniones de los demás, así mismo, este tipo de gerente debe sentir que tiene el control de todo, porque si no puede llegar a ser impaciente, intolerable e incluso impulsivo a la hora de hablar o llamar la atención a sus colaboradores. La actuación del gerente autocrático, puede alterar la armonía en el clima organizacional, ya que es dominante y desea imponer sus ideas por encima del conocimiento de sus empleados, estos últimos, pueden llegar a acumular malos sentimientos, lo cual los desmotivará y, por consiguiente, se verá reflejado en la productividad de la institución.

En segunda instancia se ubica el estilo del Gerente Consultivo, en palabras de Mora (2014) es aquel que define los objetivos y metas de la empresa en conjunto con sus subordinados, es decir, toma en cuenta las opiniones de los colaboradores y les hace participes en la selección y evaluación de alternativas de mejora. En oportunidades, este tipo de gerente es una persona insegura y constantemente debe estar consultando a sus subordinados acerca de las decisiones que deba o no tomar, lo cual no es estratégico desde el punto de la gestión.

Seguidamente se encuentra el Gerente Deliberativo, el cual se caracteriza porque comparte con sus empleados la posibilidad de estructurar metas y objetivos organizacionales, pero que en última toma la decisión de implementarlas o no, es decir este las evalúa, las monitorea, las contrasta. Este estilo de gerencia, es similar a la del Gerente Resolutivo, sin embargo, en este último la diferencia es que el gerente es el que realiza, no es el que evalúa sino el que controla. En línea similar se observa el Gerente Democrático, de acuerdo con Soto (2012) es aquel que fomenta la participación de los empleados, dejando que estos decidan obre sus funciones y tengan las suficientes competencias para tomar decisiones. Finalmente, aparece 
el Gerente Participativo, que según Valero (2012) permite que sus empleados compartan con los directivos la responsabilidad en la toma de decisiones. Aquí toma un papel fundamental la confianza, puesto que, a partir de la misma se pueden eliminar muchos prejuicios y destruir las fronteras ficticias entre las personas que ocupan cargos medios-bajos y las personas que están en la dirección. (p28).

\section{Estilos gerenciales en la generación de valor para las organizaciones}

Los diferentes estilos gerenciales, mencionados anteriormente, influyen en las directrices que siguen las compañías, así de acuerdo al estilo, o a la combinación de estos, que asuma la dirección, de igual forma se impactará el curso de la empresa en la creación de valor. De acuerdo con Peralbo (2006), la creación de valor para los propietarios de las empresas como objetivo de la gestión empresarial ha sido abordada por un nuevo enfoque de gestión, en la actualidad la generación de valor está relacionada de forma directa con la gestión de todos y cada uno de los stakeholders internos y externos de la empresa, lo que favorece la cooperación y la armonía, viéndose estos aspectos trasformados en productividad y competitividad para la empresa.

En tal sentido, los objetivos de los stakeholders internos en la actualidad, sobrepasan el alcance económico; según Young et al. (2014), cuando un empleado decide embarcarse en el camino profesional se centra en su independencia financiera, sin embargo, a través del tiempo los empleados van adquiriendo la experiencia que les permite cambiar esta preferencia netamente económica por resultados emocionales, los cuales producen en el individuo mayor satisfacción y motivación laboral, lo que contribuye enteramente a la productividad de la compañía.

Bajo esta perspectiva, un gerente de una IPS puede adelantar acciones para la compensación emocional de sus empleados, por medio de reconocimientos públicos, planes de capacitación, flexibilidad horaria, días libres, entre otras dadivas extralegales, que harán que el empleado se sienta reconocido y empoderado, es decir que crean valor en el empleado. Con relación a los stakeholders externos, específicamente los clientes, estos más allá de la calidad en los servicios de salud esperan que las IPS, brinden valor agregado en sus metodologías y calidad de atención al cliente y estilos de relacionamiento. De acuerdo con Guiteras (2012) "No hay cliente más fiel que aquel que está satisfecho con el servicio ofrecido, y no sólo con 
esto, sino también con la atención que se le ofrece después de haber adquirido dicho servicio" (p1). Por tanto, se hace necesario que los gerentes de las IPS de la región caribe colombiana, se esfuercen por estructurar estilos y códigos de atención, en donde sus clientes se sientan totalmente satisfechos y recomienden a la Institución.

Así pues, la manera en la cual el gerente se relacione con sus empleados, con los clientes, incluso con los proveedores, es decir, el estilo gerencial que este utilice, puede generar valor económico y no económico tanto para sus partes de interés, como para la empresa, en este caso para las IPS de la región Caribe colombiana, puesto que a partir de clima organizacional afable y de las relaciones redituales que se generen entre la IPS y los stakeholders, las instituciones pueden aumentar su productividad, generar mayor riqueza y posicionarse de manera sostenible en el mercado, lo anterior deriva en otro aspecto fundamental pues la gestión de calidad se pondera y esto a su vez potencia de manera integral la competitividad de la organización.

Los vertiginosos cambios en las dinámicas del mercado y las problemáticas coyunturales que emergen de un sistema de salud históricamente inestable, obliga a las IPS de la región caribe colombiana a contar con un equipo directivo capaz de afrontar los grandes retos, aprovechar las oportunidades y contrarrestar las amenazas propias del escenario donde operan. La efectividad de este proceso, depende en gran parte de la visión del gerente, el cual es el líder por excelencia, cuyo papel es guiar a los colaboradores directivos para la toma decisiones asertivas que favorezcan la calidad en la prestación de servicios de la entidad y así mismo la generación de valor para las partes de interés de la entidad.

De lo anterior, surge un cuestionamiento y es: ¿cuáles son las condiciones o criterios que deben existir para que la gestión del gerente sea considerada como buena o no? Al efectuar una revisión de la literatura, se determina que en la actualidad, la acción del gerente está influida por factores tanto endógenos como exógenos, los primeros se refieren a las condiciones personales y profesionales que el gerente ha adquirido a través de la experiencia y, los segundos, son las condiciones que no están bajo el control directo del gerente pero que siendo potenciadas de manera efectiva pueden traer resultados positivos sobre su gestión, tal es el caso del talento humano. A continuación, se detallan algunos factores endógenos y exógenos que permiten que la gestión del gerente sea efectiva y se direccione hacia la generación de valor para los Stakeholders de las IPS de objeto de estudio: 


\section{Criterios endógenos (características personales y habilidades del gerente)}

Como aspectos de interés para desarrollar competencias para el perfil de un gerente integral, han de promoverse un constructo de acciones que permitan consolidar competencias intelectuales, físicas y emocionales, las cuales han de ser trasversales a su acción profesional. De acuerdo con Amorós (2007), las habilidades de gerente se dividen en: intelectuales, físicas y emocionales. Las habilidades intelectuales, según Barrios et al. (2007) "se reconocen como el dominio complejo que el ser humano tenga de sus habilidades psíquicas y prácticas necesarias para una regulación racional" (p3). A continuación, en la figura 2, se muestran las subdivisiones de las habilidades intelectuales.

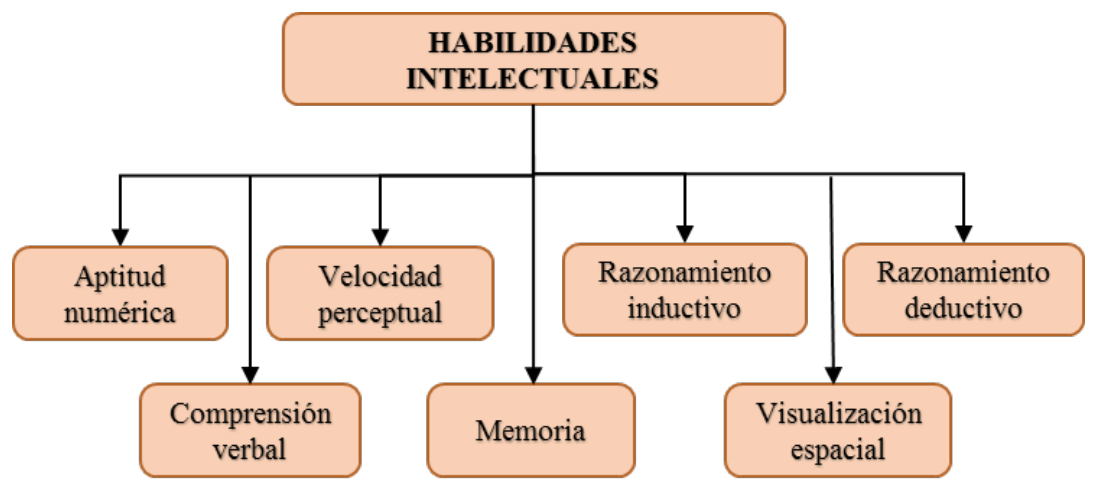

Figura 2. Habilidades intelectuales

Fuentes: Amorós (2007)

Las habilidades físicas, en palabras de Amorós (2007), "son aquellas que se necesitan para realizar tareas que demandan destreza manual," (p31) entre ellas se reconocen: factores como la flexibilidad, la fortaleza, la resistencia, el equilibrio y del vigor. Por su parte, las habilidades emocionales son un conjunto de respuestas afectivas positivas o negativas que el ser humanos puede desarrollar. Según Bisquerra (2007) "las competencias emocionales son consideradas un factor de empleabilidad, puesto que, para que las empresas sean productivas dependen de una fuerza laboral emocionalmente competente" (p1). Las habilidades emocionales están ligadas a la personalidad del individuo, dentro de estas se encuentran: la extroversión, la afabilidad la rectitud, la estabilidad emocional y, la apertura a la experiencia. 
En síntesis, Aguirre (2007) manifiesta que el gerente consolidar tres habilidades gerenciales básicas, la cuales llevan de manera intrínseca las características vistas hasta este punto, estas son: habilidades técnicas, humanas y conceptuales. Las habilidades técnicas, se refiere a los conocimientos y las experiencias que el gerente tenga en determinados procesos. Las habilidades humanas, se enfocan en la manera en la que el gerente interactúa efectivamente con la gente. Las habilidades conceptuales se tratan de la formulación de ideas y, la resolución de problemas en forma creativa (p1).

\section{Criterios exógenos que influyen en la acción del gerente}

Existen factores que no dependen propiamente del gerente, pero que, aun así, interfieren en su praxis profesional; entre estos se encuentran aspectos económicos, políticos, tecnológicos, y aquellos que tienen que ver con la fuerza laboral. Los factores económicos, se refieren a los cambios en las dinámicas financieras y económicas, como el aumento de la inflación, el incremento de la carga tributaria, las recesiones económicas, entre otras. Se prevé que el gerente no puede, por ejemplo, controlar el precio de los insumos, pero si efectuar acciones estratégicas para priorizarlos. Los factores políticos según Parada (2013) "son aquellos asociados a las decisiones de los gobiernos locales, como es el caso de las políticas fiscales" (p1). Estos factores pueden afectar en gran medida a las IPS públicas, puesto que de las decisiones políticas dependen su continuidad y estabilidad de tal manera que no puede combatir el sistema, pero si transformar estos retos en grandes oportunidades de crecimiento.

De acuerdo con Taylor (2014) la tecnología afecta a los entornos empresariales, puesto que, a medida que la tecnología avanza, una empresa se ve obligada a mantener el ritmo. En muchas ocasiones las IPS, no cuentan con el capital económico suficiente para adquirir equipos médicos de ultima tecnología, lo cual sugiere que el gerente planee la manera de adquirirlos, o de cubrir la ausencia de los mismos con otros equipos menos costos combinados con capital humano cualificado, de este modo, mejorar la calidad de servicios. Con relación al capital humano, Bracca (2011) manifiesta que el gerente, debe ser "un estratega en relaciones humanas para vincular a los clientes tanto internos como externos y los distintos procesos que se cumplen en la empresa, así como promover a líderes de los medios para el mejoramiento de sus capacidades" (p1). El capital humano permite que las IPS de la región caribe creen ventajas competitivas comprendidas en los conocimientos tácitos o explícitos, los cuales son difíciles de imitar y, por tanto, generan valor para la empresa. 
Los anteriores factores no son controlables por el gerente, pero si son modificables y aprovechables si este utiliza sus capacidades personales y habilidad gerenciales de la manera adecuada. El gerente más que ser un administrador, debe ser un gestor de cambio de relaciones interpersonales, con miras al crecimiento sostenido a través de la generación de valor económico, emocional, relacional, entre otros, para la entera satisfacción de los sectores relacionados.

\section{Habilidades gerenciales tradicionales vs habilidades gerenciales contemporáneas}

Los cambios que se han generado en el mercado, sugieren una trasformación radical de los paradigmas administrativos tradicionales, a partir de este proceso, el gerente puede mejorar sus actuaciones, adecuándose de manera armónica a los requerimientos actuales. Es importante resaltar, que el gerente ha tenido que mutar sus funciones históricamente rígidas y autocráticas, a funciones flexibles y horizontales, donde se permita escuchar la opinión de los demás y a raíz de ello pueda no solo concebir la realidad de la empresa, sino mejorarla. Otros de los cambios que se han suscitado en el estilo del gerente contemporáneo comparado con el gerente tradicional, es que este anteriormente se posicionaba en la cúspide de la estructura organizacional, pero en la actualidad, debe ubicarse en el centro de la organizacional, pues esta posición le permite mirar de manera circular y holística todo lo que ocurre en la empresa.

El gerente anteriormente no aceptaba errores, sin embargo, actualmente se permite fallar, porque es precisamente en el fracaso donde se pueden hallar las mejores maneras de evolucionar; así mismo, el gerente se basaba en la información del pasado para gestionar el presente, pero, el gerente actual, visualiza los escenarios futuros deseables y posibles, para desarrollar acciones presentes que le lleven a conseguir lo proyectado. Finalmente, el gerente tradicional se reconocía por ser cerrado y monologuista, pero en la actualidad este debe ser dinámico, participativo, abierto a los demás, motivador y estimulador, además, un líder por excelencia con capacidad para hacer sinergia con todos los procesos con el fin de generar resultados que agreguen valor a la institución. Hasta este punto, se ha observado cómo el gerente ha tenido que mutar su actuación en aras de hacer frente a los requerimientos de las dinámicas empresariales actuales; los estilos de gestión bajo esta lógica han demostrado ser altamente efectivos, porque consideran algo que tal vez no se pensaba en el pasado: generar procesos de interacción con todos los sectores que deben interactuar. 


\section{Metodología}

El presente estudio se fundamenta en un tipo de investigación descriptiva/documental, método cualitativo el artículo se efectúa a partir del método cualitativo de investigación, en el cual, según Hernández et al., (2010), "el investigador ve el escenario y a las personas en una perspectiva holística; las personas, los ambientes o los grupos no son reducidos a variables, sino considerados como un todo" (p9). En función de lo descrito se procedió con el análisis de manera integral de la incidencia de los estilos gerenciales, en la creación de valor; se estudia este fenómeno en su contexto natural, es decir no se modificarán las variables, sino que se describirán en su contexto original.

Asimismo se procedió con la selección de la técnica a través del diseño de una entrevista estructurada, conjuntamente con la teoría fundamentada, llevar a efecto el análisis entre la percepción de los gerentes y los datos obtenidos de fuentes de información científica, que sustentan el estudio de las variables estilos gerenciales y generación de valor organizacional , para determinar qué significado simbólico tienen los fenómenos y cómo interactúan unos con otros, buscando con ello un análisis reflexivo sobre la base de las teorías expuestas. Según Rodríguez et al., (1996), citado por Mayol (2014) "El enfoque del que parte la teoría fundamentada es el de descubrir teorías, conceptos, hipótesis y proposiciones partiendo directamente de, los datos, y no de supuestos a priori, de otras investigaciones o de marcos teóricos existentes" (p24). Al optar por este tipo de estudios, se busca que la información sea abordada más no manipulada para lograr una conceptualización mucho más cercana y natural conforme a la realidad.

\section{Análisis de resultados}

Los resultados de las técnicas aplicadas permitieron determinar que los estilos gerenciales tienen una influencia directa en la generación de valor de las IPS de la región caribe, en la medida en que estas, a su vez, creen valor para las partes de interés. En tal sentido, se ratifica que las empresas deben ser concebidas como un "todo integral" en donde cada uno de sus elementos constitutivos, llámense proveedores, clientes o empleados son indispensables para la operación y el crecimiento sostenido de la empresa en el mercado. 
Desde esta perspectiva, la interacción con los gerentes de 12 IPS de la Costa Caribe colombiana arrojo resultados de coincidencia donde se dimensiona el interés de afianzar un estilo gerencial participativo a su gestión. Estilo el cual, según Ruiz (2012) "proyecte seguridad y confianza a sus trabajadores", puede motivar y empoderar a su fuerza laboral, lo que se traduce al aumento de la productividad de la misma, posicionamiento en el mercado y auge de la gestión de calidad. En consecuencia, se prevé desde la perspectiva teórica y funcional que si el gerente promueve dentro de su sistema de gestión formas de atención al cliente personalizadas y diferenciales, lo más seguro es que los usuarios de los servicios se sientan fidelizados por la institución, decidan regresar a la entidad y recomendarla, pues confían en sus procesos y se sienten respaldados por la gestión de mejoramiento.

A partir de lo descrito, se procede a relacionar la importancia que se acredita a las competencias gerenciales para promover la generación de valor organizacional, por lo que las capacidades del gerente en identificar y prever de forma objetiva los riesgos y oportunidades que puede enfrentar la IPS, es considerados por los gerentes entrevistados como parte de la estrategia que permite optimar y equilibrar las relaciones entre las diferentes expectativas de los sectores relacionados (stakeholders), por lo que sus potencialidades y competencias permitirá plantear estrategias que aseguren la generación de valor para empleados, clientes, proveedores, accionistas y, en general la sociedad, a partir de una efectiva concepción sobre las implicaciones de un gerente efectivo que busca la participación, la inclusión, la innovación, el conocimiento, desde el contexto delas IPS, así como la posibilidad de invertir en bienes y activos intangibles para el desarrollo de ventajas competitivas que permitan maximizar el valor económico de la entidad, y el aseguramiento de los procesos.

Como componente estratégico obtenido de los resultados, se especifica los planteamientos realizados por los gerentes de la IPS, ante la necesidad de viabilizar estilos gerenciales que de forma integral permitan impulsar y proyectar el direccionamiento de las instituciones objeto de estudio. Por lo cual, la detección de debilidades presentadas e identificados como indicadores de las variables como lo son: la gestión de recursos humanos, asertividad en la definición de objetivos, efectividad en la gestión de materiales e insumos y en general, así como la minimización de los gastos fijos de operación, considerados desde la perspectiva de las unidades de análisis como los principales componentes que de no ser gestionados de forma eficientemente debilitan el sector. En función de ello, se identifica que desde el contexto 
de las IPS, los perfiles y estilos gerenciales ameritan ser impulsados para consolidar una dirección efectiva capaz de generar importantes transformaciones que contribuyan a incrementar el grado de participación y cobertura de la gestión de las IPS y por ende de los servicios y productos que en el marco de la generación de valor.

\section{Conclusiones}

El determinar cómo los estilos y habilidades gerenciales influyen en la generación de valor en las IPS de la región Caribe, permitió concluir la gestión de las IPS, así como la necesidad de su transformación responden, no sólo de requerimientos propios de las dinámicas empresariales contemporáneas, sino también de las capacidad y estilos gerenciales, por lo cual su influencia sobre la generación de valor en la gestión de las IPS, frecuentemente es condicionada a los diversos estilos y habilidades gerenciales.

En función de ello, se considera como aporte del estudio la identificación desde el contexto de las IPS las características propias de cada uno de los estilos gerenciales y las competencias que han de consolidar características distintivas para la generación de valor. En consecuencia, resulta determinante la captación de un perfile gerencial integro, capaz de gestionar de manera efectiva las instituciones objeto de estudio. De tal manera, que optimar los procesos de selección de gerentes para el sector considerando el perfil de competencias cognitivas, técnicas y humanas donde la experiencia, opinión e interacción con todos los miembros de la organización se consideren como elementos diferenciadores que impactan a lo interno: con cada uno de sus colaboradores; y a los externo con todos los sectores relacionados, lo cual permitirá proyectar una visión más amplia del contexto donde se desenvuelve para proyectarse de manera estratégica hacia el futuro.

En líneas generales, como aporte significativo para el sector se fundamenta a través del estudio que el estilo gerencial se constituye en la principal fortaleza mediante la cual se enfrentan los riesgos e incertidumbres, así como se generan condiciones para adaptarse a las transformaciones sociales impuestas por un entorno global que impacta a las IPS, en consecuencia la generación de valor en el proceso organizacional de las IPS, debe manejarse de manera íntegra con la disponibilidad de activos tangibles e intangibles cuya gestión sea alcanzada bajo estándares de eficiencia y eficacia. 


\section{Referencias}

Aguirre, M. A. (2007). Habilidades directivas o Habilidades gerenciales. Recuperado de: http://www.degerencia.com/tema/habilidades_gerenciales.

Amorós, E. (2007). Comportamiento organizacional: en busca del desarrollo de ventajas competitivas. Perú: Editorial Universidad Católica Santo Toribio Mogrovejo, Escuela de Economía.

Argandoña, A. (2011). La teoría de los Stakeholders y la creación de valor. Recuperado de: http://www.iese.edu/research/pdfs/DI-0922.pdf.

Barrios, M. A.; Lorenzo, E; Bacallao, I. y Serrano, L. M.; (2007). Desarrollo de las habilidades intelectuales en las ciencias Morfológicas. Archivo Médico de Camagüey, 11() Recuperado de http://www.redalyc.org/articulo.oa?id=211118015018.

Bisquerra, R. y Pérez, N. (2007). Las competencias emocionales. Educación XXI. 10. 61-82. Recuperado de: http://www.ub.edu/grop/catala/wp-content/uploads/2014/03/Las-competencias-emocionales.pdf.

Bracca, C. (2011). El gerente del talento humano. Recuperado de: http://lagerenciamodernaenlasorganizaciones.blogspot.com.co/2011/01/el-gerente-del-talento-humano.html.

Guiteras, X. (2012). La importancia de un buen servicio de atención al cliente. Recuperado de: http://marketingyconsumo.com/la-importancia-de-un-buen-servicio-de-atencion-alcliente.html.

Hernández, R.; Fernández, C. y Baptist,a L. (2010). Metodología de la investigación. Recuperado de: https://www.esup.edu.pe/descargas/dep_investigacion/Metodologia\%20de\%20 la\%20investigaci\%C3\%B3n\%205ta\%20Edici\%C3\%B3n.pdf.

Larocca, H.A. (2009). (s/f) Estilos Gerenciales para el Nuevo Milenio. Recuperado de: https:// clea.edu.mx/biblioteca/Estilos\%20gerenciales\%20para\%20el\%20nuevo\%20milenio.pdf.

Mayol, A. (2014). Metodología de la Investigación Cualitativa. Recuperado de: http://www. albertomayol.cl/wp-content/uploads/2014/03/Rodriguez-Gil-y-Garcia-Metodologia-Investigacion-Cualitativa-Caps-1-y-2.pdf. 
Ministerio de la Protección Social (MPS) (2010). ¿Cómo es el POS vigente en Colombia? Disponible en: http://www.pos.gov.co/Paginas/default.aspx

Ministerio de la Protección Social (MPS) (2011). Registro Especial de Prestadores de Servicios de Salud. Disponible en: http://www.minproteccionsocial.gov.co/consultaInformación/default.aspx

Mora, J. (2014). Estilos gerenciales. Recuperado de: http://iupsm-jemp.blogspot.com.co/2009/08/ estilos-gerenciales.html.

Parada, P. (2013). Análisis PESTEL, una herramienta de estrategia empresarial de estudio del entorno. Recuperado de: http://www.pascualparada.com/analisis-pestel-una-herramienta-de-estudio-del-entorno/

Peralbo, A. C. (2006). Relación entre los stakeholders y el valor de las empresas (Doctoral dissertation, Universidad de Córdoba).

Ruiz, P. A. (2012). Las Habilidades Gerenciales y su Importancia en la Dirección Empresarial. Recuperado de: http://www.revista-mm.com/ediciones/rev78/admin_habilidades.pdf.

Solys, G. A. P., Morales, M. B., y Medina, W. M. G. (2016). Los estilos de liderazgo y su influencia en el desarrollo empresarial: caso Pymes de la provincia de Tungurahua-Ecuador. Revista PUCE, (103).

Soto, B. (2012). El liderazgo democrático. Recuperado de: http://www.gestion.org/recursoshumanos/liderazgo/45862/el-liderazgo-democratico/

Taylor, H. (2014). Factores que afectan al entorno económico de una empresa. Recuperado de: http://www.ehowenespanol.com/factores-afectan-entorno-economico-empresa-lista_116299/.

Valero, C. (2012). La Gerencia de la desconfianza. Palibrio.

Young, M., Edmunds \& Otros (2014). The tenuous relationship between salary and satisfaction. Recuperado de: http://www.aabri.com/manuscripts/141782.pdf 